\title{
Contribución de Pheidole fallax y Ectatomma ruidum (Hymenoptera: Formicidae) en la dispersión y germinación de semillas en áreas rehabilitadas de la mina de carbón del Cerrejón, Colombia
}

\author{
Bleydis Paola Gutierrez-Rapalino ${ }^{1} \&$ Yamileth Domínguez-Haydar ${ }^{1}$ \\ 1. Grupo de Investigación Biodiversidad del Caribe colombiano. Programa de Biología, Facultad de Ciencias \\ Básicas, Universidad del Atlántico. Ciudadela Universitaria, Km 7-Vía Puerto Colombia. Barranquilla, Colombia; \\ bleydis1804@gmail.com, yamilethdominguez@mail.uniatlantico.edu.co
}

Recibido 30-V-2016. Corregido 08-XI-2016. Aceptado 07-XII-2016.

\begin{abstract}
Contribution of Pheidole fallax and Ectatomma ruidum (Hymenoptera: Formicidae) to the dispersion and germination of seeds in rehabilitated areas of the Cerrejón coal mine, Colombia. Seed dispersal is a key process in the re-establishment of vegetation on reclaimed lands. The aim of this study was to determine the contributions of the ant species Ectatomma ruidum and Pheidole fallax to seed dispersal and germination on reclaimed lands in the Cerrejón coal mine, Colombia. Four 6 x $40 \mathrm{~m}$ plots were installed in each area, in which the number of nests by species was counted and distances between closest nests of each species were measured. Five of the nests counted were chosen for seed extraction, and a nearby control sample was taken from each nest. In total, 295 nests were counted: 59 nests during the dry season and 236 nests during the rainy season, of which 84 belonged to $P$. fallax and 211 belonged to E. ruidum. A total of 21304 seeds were found in nests and control samples, of which 19349 were obtained from $P$. fallax nest refuse, and 318 were obtained from E. ruidum nests; 597 seeds were found in control samples. The number of seeds extracted from nests was significantly higher than the number of seeds removed from control samples. There was a significant difference between the number of seeds removed by $P$. fallax and the number of seeds removed by E. ruidum. The seed germination percentage from nests was higher than the percentage from seeds in control samples. In the case of $P$. fallax, the germination percentage was lower in control samples and higher in nests and refuse. The results suggest that the ant species $E$. ruidum and $P$. fallax may contribute to seed dispersal and re-establishment of vegetation in areas where they were found. These ants may thus significantly contribute to restoration processes in areas degraded by mining. Rev. Biol. Trop. 65 (2): 575-587. Epub 2017 June 01.
\end{abstract}

Key words: myrmecochory, nests, restoration, refuse piles, propagules.

La dispersión de semillas es el movimiento de los propágulos fuera del área de influencia de la planta madre; en este proceso los animales cumplen un papel importante como vectores (Howe \& Smallwood, 1982). Entre los insectos se ha destacado el aporte de las hormigas como dispersores de semillas en la región Neotropical (Pizo, Passos, \& Oliveira, 2004; Christianini, Mayhé-Nunes, \& Oliveira, 2007, 2012). En la mirmecocoria las hormigas transportan las diásporas a una distancia considerable de la planta madre sin afectar el embrión, les permiten la protección frente a depredadores, la disminución en la competencia con los congéneres y les brindan además la oportunidad de colonizar otras áreas (Beattie, 1985).

Las hormigas como postdispersoras reorganizan los bancos de semillas y pueden aumentar las tasas de germinación (Delabie, Ospina, \& Zabala, 2003; Giladi, 2006; Farnese, Mângia, \& Fonseca, 2011; Escobar-Ramírez, Duque, Henao, Hurtado-Giraldo, \& Armbrecht, 2012). Sin embargo, la propagación depende en cierta medida de las características de las semillas 
o de las preferencias alimenticias de las hormigas. Varias investigaciones han demostrado que la presencia de apéndices, como arilo, o de una estructura carnosa llamada eleosoma podría aumentar la dispersión (Beattie, 1989; Pizo et al., 2004; Salas, Ybarra, Moreno, Rivas, \& Pérez, 2010). Incluso, las semillas que contienen mayor cantidad de lípidos en su parte carnosa suelen presentar una mayor tasa de remoción (Christianini et al., 2007).

Las semillas removidas por las hormigas, son llevadas a sus nidos donde son consumidas las estructuras carnosas y muchas veces la semilla es dejada intacta. Esta situación podría aumentar la supervivencia y el éxito germinativo (Christianini et al., 2007), ya que los nidos son sitios enriquecidos en nutrientes, en los que las semillas encuentran micrositios adecuados que pueden favorecer su germinación y el establecimiento de las plántulas (Leal, Wirth, \& Tabarelli, 2007). Desde este punto de vista, esta función resulta beneficiosa en áreas en las cuales se realizan procesos de restauración, ya que podrían contribuir con la recuperación de la capa vegetal afectada por actividades humanas de alto impacto como la minería de carbón a cielo abierto.

En la mina de carbón del Cerrejón (La Guajira, Colombia) desde hace más de dos décadas se han realizado acciones de rehabilitación ecológica. De acuerdo con DomínguezHaydar y Armbrecht (2011) la aplicación de estos planes de restauración ha permitido el restablecimiento de la fauna de hormigas y la recuperación de algunas funciones ecosistémicas; en particular, encontraron que las especies Ectatomma ruidum (Roger, 1861) y Pheidole fallax (Mayr, 1870) fueron las hormigas que más contribuyeron a la remoción y dispersión de semillas.

Resultados similares encontrados por Wilson (2003), Zelikova y Breed (2008), Santamaría, Armbrecht y Lachaud (2009 a), y Lôbo, Tabarelli y Leal (2011) muestran que estas hormigas remueven más del $50 \%$ de las semillas que les son ofertadas en los experimentos de remoción. Además, Zelikova y Breed (2008) indican que la remoción de semillas por estas hormigas es espacialmente diferente, E. ruidum realiza esta labor hacia áreas abiertas como potreros, mientras que $P$. fallax lo hace hacia lugares con mayor cobertura vegetal.

Pese a que E. ruidum y $P$. fallax han sido reconocidas como buenas dispersoras de semillas, pocas referencias detallan qué sucede con las semillas llevadas a los nidos; es decir, si son o no consumidas y cómo es la tasa de germinación respecto a las que no son removidas. Este estudio cobra importancia si se tiene en cuenta que estas hormigas se encuentran asociadas a sitios en procesos de restauración, donde el papel como dispersores podría contribuir al restablecimiento de la capa vegetal. Por ello, el objetivo de este trabajo fue evaluar la contribución de las hormigas E. ruidum y P. fallax en la remoción y germinación de semillas en áreas rehabilitadas en la mina del Cerrejón.

\section{MATERIALES Y MÉTODOS}

Área de estudio: Este trabajo fue realizado en la mina de carbón a cielo abierto del Cerrejón, localizada en el departamento de $\mathrm{La}$

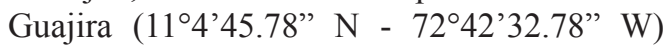
(Fig. 1), al noreste de Colombia (Santamaría, Domínguez Haydar, \& Armbrecht, 2009 b; Gualdrón, 2011). Las zonas climáticas en el área corresponden a las categorías de cálido seco y cálido muy seco. Se presentan dos períodos lluviosos; el primero en abril y mayo, el segundo entre octubre y noviembre, con una precipitación media anual de $840 \mathrm{~mm}$, una temperatura media anual de $27.5^{\circ} \mathrm{C}$ y una elevación que fluctúa entre $220 \mathrm{~m}$ y $240 \mathrm{~m}$. La formación vegetal corresponde a las zonas de matorral desértico, bosque subxerofítico y bosque seco tropical (Gualdrón, 2011).

La mina utiliza un modelo de rehabilitación ecológica que consta de tres etapas: adecuación geomorfológica de las tierras, estabilización de los suelos con pastos y la revegetación con especies nativas (Gualdrón, 2011). Producto de esta labor realizada por la mina, podemos encontrar áreas con diferentes tiempos de haber iniciado el proceso de rehabilitación en un gradiente que alcanza más de 20 años 


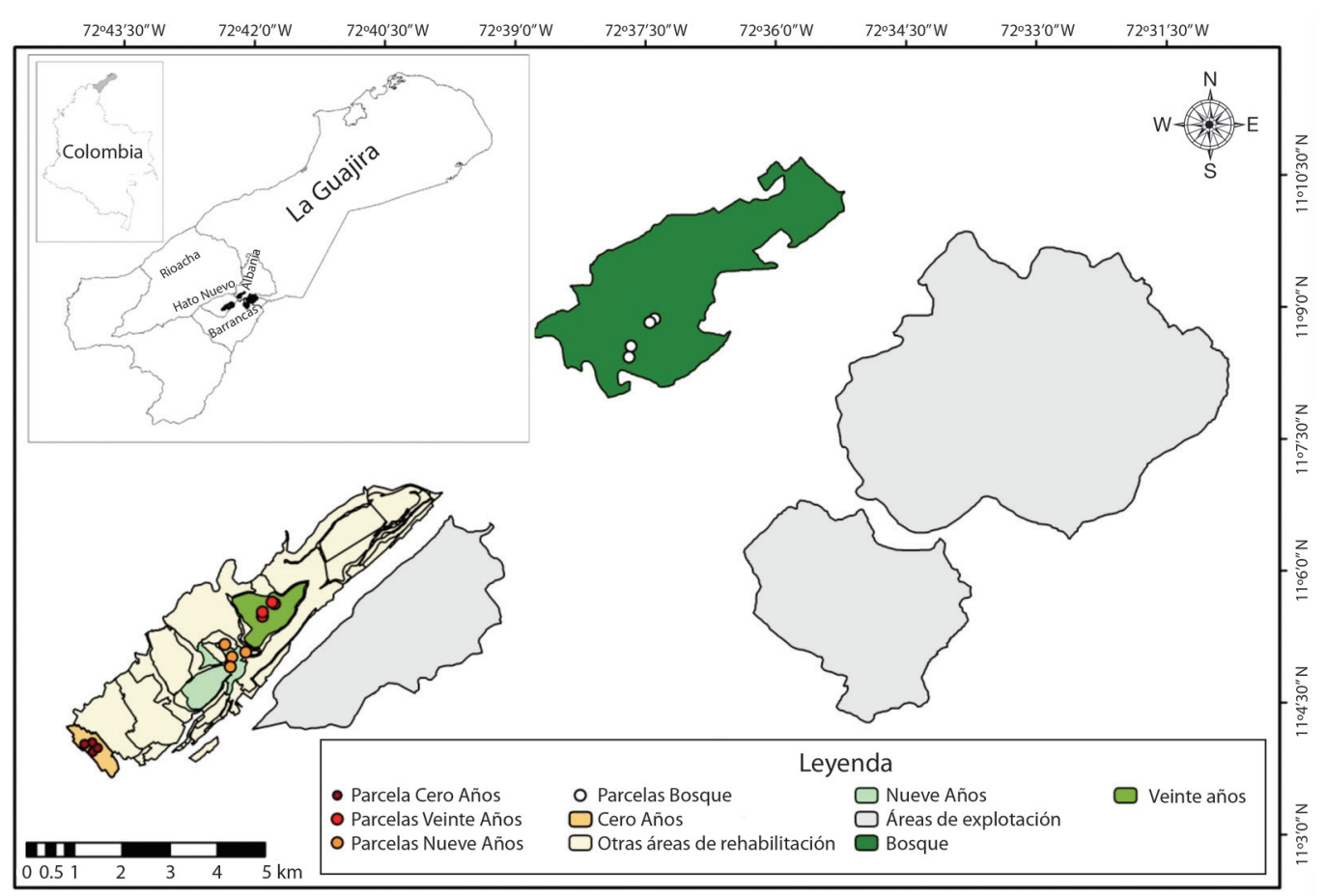

Fig. 1. Área de estudio en donde se muestran las áreas de rehabilitación, las zonas de explotación de carbón actual y el bosque de referencia en la mina de carbón el Cerrejón, Guajira-Colombia.

Fig. 1. Study area showing rehabilitation areas of current coal exploitation and the forest site in the Cerrejon coal mine, Guajira, Colombia.

(más detalles en Gualdrón (2011) y Domínguez-Haydar y Armbrecht (2011)). En los alrededores de la mina se encuentran fragmentos de bosque seco tropical, los cuales no han sido intervenidos con minería y forman parte de la zona de compensación y conservación.

Diseño de muestreo: Se realizaron dos muestreos durante el 2014, uno en la época seca (marzo) y otro en la época de lluvia (diciembre). Para determinar la presencia de $P$. fallax y E. ruidum, se realizó un premuestreo en áreas con uno, nueve y veinte años de iniciado el proceso de rehabilitación y un bosque no intervenido en minería. En el sitio de un año no se encontraron estas hormigas, por tanto, se seleccionó el resto de áreas (nueve años, veinte años y bosque no intervenido); en cada una de ellas se instalaron cuatro parcelas de 6 x $40 \mathrm{~m}$, en ellas se marcaron y contabilizaron los nidos presentes en cada parcela. De cada especie se extrajeron cinco nidos al azar por parcela con su respectivo suelo control, para este se verificó que no hubiera actividad superficial de macrofauna y se tomó a un metro de distancia del nido. Para asegurar la independencia entre nidos se comprobó que la distancia mínima entre los nidos de $P$. fallax fuese de dos metros, a través de excavaciones previas se determinó que nidos con una separación de aproximadamente $1 \mathrm{~m}$ no estaban comunicados (Obs. pers); en cuanto a E. ruidum, esta especie es considerada monodómica (Breed, Abel, Bleuze, \& Denton, 1990; Breed, McGlynn, Stocker, \& Klein, 1999), no obstante, la distancia mínima entre nidos fue de $1 \mathrm{~m}$.

Los nidos fueron transportados en bolsas plásticas al laboratorio de Biología General de la Universidad del Atlántico para la extracción de las semillas presentes. Las semillas encontradas en las diferentes categorías: nidos, controles (suelo sin nidos) y basureros de ambas 
especies de hormigas fueron identificadas al compararlas con las semillas de las especies de plantas registradas por Jaimes y Moreno (2014) en áreas rehabilitadas y bosques del Cerrejón. También fueron utilizadas las guías de identificación de semillas propuestas por Torres, Zapote y López (2013 a, b). Las semillas se clasificaron en tres categorías: Consumidas, intactas y secas.

Experimento de germinación: Se determinó la viabilidad de las semillas intactas extraídas de los nidos, controles y basureros de $P$. fallax y E. ruidum, se colocaron a germinar 895 semillas. Cinco semillas de cada tipo fueron colocadas en recipientes plásticos con algodón humedecido y se regaron cada dos días con $2 \mathrm{~mL}$ de agua. El experimento tuvo un período de duración de 30 días y se consideró como éxito germinativo las semillas que presentaron emergencia de la radícula (Leal, 2003). Se tomó en cuenta el origen de las semillas (especie de hormiga, área y a cuál categoría del suelo pertenecía: nido, control o basurero).

Experimento de interacción natural hormigas-semillas: Para determinar el tipo de interacción de las hormigas con las semillas se realizaron tres experimentos. En junio de 2015 se recolectaron ejemplares de ambas especies con las que se establecieron cuatro nidos artificiales, dos de $P$. fallax y dos de E. ruidum; estos nidos permanecieron en el laboratorio de zoología de la Universidad del Atlántico por un período de 30 días. Los nidos artificiales fueron construidos de acuerdo con lo propuesto por Pizo y Oliveira (2001), estos consistieron en una caja plástica (largo $=40 \mathrm{~cm}$, ancho $=30$ $\mathrm{cm}$ y alto $=12 \mathrm{~cm}$ ) donde se recreó un tubonido, el cual consistió en un tubo de ensayo de $8 \mathrm{~cm}$ de largo y $1 \mathrm{~cm}$ de diámetro. En parte final del tubo se mantuvo un $1 \mathrm{~cm}$ de agua retenida con un algodón para tener una fuente de humedad, el tubo fue cubierto en la parte exterior con papel aluminio para aislarlo de la luz. Las hormigas en el período de adaptación fueron alimentadas con miel o una mezcla de una yema de huevo crudo, 12 g azúcar y $25 \mathrm{~g}$ miel, ocasionalmente se les ofreció larvas de otros insectos. Los nidos de P. fallax contenían 11 soldados y 33 obreras, el nido uno, y 7 soldados y 24 obreras, el nido dos. Para $E$. ruidum, el nido uno tenía 27 obreras y el nido dos 21 obreras.

Los experimentos se realizaron entre junio y julio 2015 en el laboratorio de zoología de la Universidad del Atlántico. En el primero se evaluó la preferencia de las hormigas por el tamaño de la semilla, para ello en cada nido se ofrecieron tres semillas de diferentes tamaños: grandes $(6 \mathrm{~mm}$ a $1 \mathrm{~cm})$, medianas $(2.6 \mathrm{~mm}$ a $5.5 \mathrm{~mm})$ y pequeñas $(1.2 \mathrm{~mm}$ a $2.5 \mathrm{~mm})$, las tres semillas fueron ofrecidas al mismo tiempo para determinar la preferencia, estas se colocaron sobre un papel de color blanco para facilitar la visualización. Este experimento fue realizado 10 veces por nido y en total se obtuvo 20 observaciones por cada una de las especies.

El objetivo del segundo y tercer experimento fue observar el comportamiento de las hormigas con las semillas al ser llevadas al tubo-nido, para observar qué sucedía con la semilla que era ingresada, se retiraba el papel aluminio. En el segundo experimento se ofreció una semilla de la especie más abundante encontrada en los nidos de cada especie de hormiga; en el tercer experimento se ofreció a ambas especies una semilla de olivo (Capparis sp., semilla con estructura carnosa). Cada experimento se repitió 10 veces en cada nido y se utilizaron los mismos individuos. Se contabilizó las veces que la semilla fue ingresada al tubo-nido, las que no fueron removidas, las que fueron llevadas al nido y luego trasladadas al basurero y las que fueron removidas pero no ingresaron al tubo-nido. Además, se observó si estas semillas fueron consumidas total o parcialmente.

Para establecer si las especies $P$. fallax y $E$. ruidum contribuyen a la remoción de semillas, se evaluó si existen diferencian significativas entre las semillas encontradas en los nidos y controles de E. ruidum y en nidos, basureros y controles de $P$. fallax. Para ello se utilizó la prueba de Wilcoxon. Para determinar si existen diferencias en el número de semillas removidas 
por P. fallax y E. ruidum se utilizó el test de U-Mann-Whitney para comparación de pares de muestras. Además, para establecer si existen diferencias en la remoción de semillas de cada hormiga en las distintas áreas, se utilizó una prueba de Kruskall-Wallis, acompañado del test de comparación de pares de muestras de $\mathrm{U}$ Mann-Whitney. Estos análisis fueron realizados con el programa Past 3.06 (Hammer, 2015).

Con el fin de determinar si ambas especies de hormigas tienen preferencia por algunas de las semillas encontradas en sus nidos se utilizó el índice del valor indicador (IndVal) propuesto por Dufrene y Legendre (1997). Este índice utiliza una matriz de abundancia de las semillas encontradas en los nidos de cada especie de hormiga para evaluar el grado con el que cada una exhibe especificidad (presencia única de un tipo de semilla en sus nidos) y fidelidad (frecuencia de ocurrencia de un tipo de semilla en sus nidos). Para realizar este análisis se usó el paquete labdsv y fue ejecutado con el programa R versión 3.1.1 (R Development Core Team, 2014).

Por último, con el fin de evaluar si existen diferencias entre el porcentaje de germinación de semillas entre los nidos y los controles de E. ruidum y nidos, basureros y controles de $P$. fallax se utilizó la prueba de Kruskal-Wallis.
Estos análisis fueron realizados con el programa Past 3.06 (Hammer, 2015).

\section{RESULTADOS}

Abundancia de semillas: Se encontró un total de 21304 semillas, de las cuales 318 fueron obtenidas de los nidos de E. ruidum, 3221 en nidos de $P$. fallax y 17128 en los basureros de esta última. En los controles (suelo sin presencia de nidos) se encontraron 597 semillas. El $84.43 \%$ de las semillas estaban intactas, el $8.73 \%$ secas y un $6.84 \%$ estaban consumidas. El mayor número de semillas consumidas e intactas se encontraron en los basureros de P. fallax, con $7.4 \%$ y $85.4 \%$, respectivamente. En cuanto a las épocas, las semillas consumidas fueron mayores en la época seca (11.6\%) y las semillas intactas fueron mayores en la época de lluvias (89.7 \%). Al comparar los nidos de ambas especies encontramos que $P$. fallax presenta un mayor número de semillas intactas independientemente de la época (Fig. 2).

El número de semillas removidas por ambas especies de hormigas en la época de lluvia fue significativamente mayor en comparación con la época seca según la prueba de Kruskal-Wallis $(\mathrm{H}=6.846 ; \mathrm{P}<0.01)$. Al considerar la prueba pareada de Wilcoxon

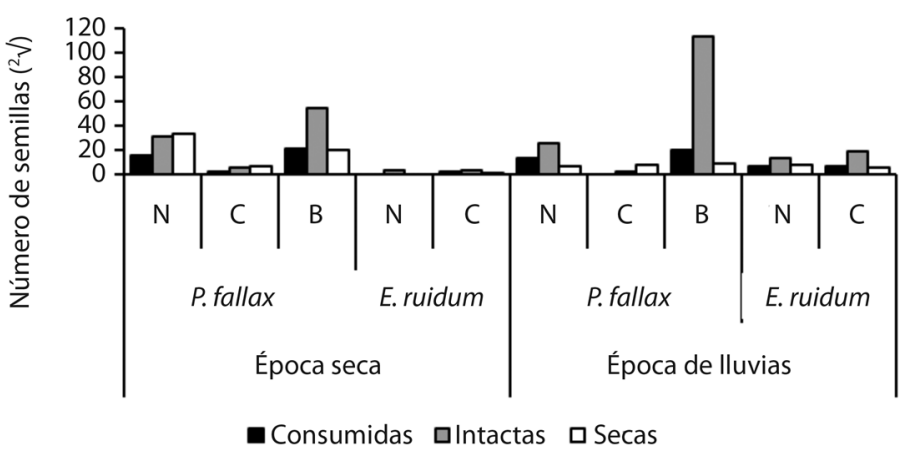

Fig. 2. Número de semillas encontradas por categoría de suelo, según época y especie. Los datos de abundancia de semillas fueron transformados a raíz cuadrada. Nido (N), Control (C) y Basurero (B).

Fig. 2. Number of seeds found in different soil categories, taking into accounts the season and species. Data seed abundances were square root transformed. Abbreviations: Nest (N), Control (C) and Dump (B). 
se determinó que existen diferencias en el número de semillas encontradas en nidos y controles de E. ruidum $(\mathrm{Z}=-2.011 ; \mathrm{P}<0.05)$ siendo mayor la presencia de semillas en los nidos. En cuanto a las áreas se observó que $E$. ruidum no presentó diferencias en la remoción de semillas por áreas $(\mathrm{H}=1.101 ; \mathrm{P}>0.05)$.

Se demostró que el número de semillas encontradas en los nidos de $P$. fallax fue significativamente mayor que en los controles $(\mathrm{Z}=-2.55 ; \mathrm{P}<0.05)$, mientras que el número de semillas encontradas en los basureros fue mayor a la encontrada en nidos $(\mathrm{Z}=-2.54 ; \mathrm{P}<$ $0.05)$ y controles $(Z=-3.18 ; P<0.01)$. Existen diferencias entre el número de semillas removidas por áreas $(\mathrm{H}=15.19 ; \mathrm{P}<0.01)$. El área de 20 años presentó diferencias con las áreas de bosque $(\mathrm{U}=97.5 ; \mathrm{P}<0.01)$ y nueve años $(\mathrm{U}=$ 138.5; $\mathrm{P}<0.01)$ según la prueba de U-MannWhitney, en esta última área la remoción fue mayor. En cuanto al bosque, éste no presentó diferencias con el área de nueve años $(U=208$; $\mathrm{P}>0.05)$. El número de semillas removidas por $P$. fallax es significativamente mayor a las removidas por E. ruidum $(\mathrm{Z}=-2.37 ; \mathrm{P}<0.05)$.

Tipos de semillas encontradas en los nidos de $\boldsymbol{P}$. fallax y $\boldsymbol{E}$. ruidum: El total de semillas intactas encontradas en nidos, basureros y controles fue de 17199 , las cuales se encontraron distribuidas en 23 tipos (morfoespecies). P. fallax removió 16665 semillas (3 141 para la época seca y 13524 para la época de lluvia). Durante la época seca se removieron 10 tipos de semillas, observándose la mayor remoción en el área de 20 años, siendo la semilla Desmanthus sp. 1, la más encontrada en los nidos de esta especie. Para la época de lluvias fueron encontradas 15 tipos de semillas en nidos y basureros. Bastardia sp. 1 fue la especie que presentó mayor abundancia (Cuadro 1, Fig. 3).

En cuanto a E. ruidum se encontraron 534 semillas intactas, 24 para la época seca y 510 para la época de lluvia. Esta hormiga removió solo un tipo de semilla para la época seca (Fabaceae sp. 1), para la época de lluvia se observaron 11 tipos de semillas, Panicum sp. 1 fue la de mayor abundancia (Cuadro 2, Fig. 3).

En relación a las semillas encontradas en los nidos de ambas especies, el índice de valor indicador mostró que $P$. fallax tiene preferencia por las semillas de Desmanthus sp. $1(\mathrm{R}=$ 0.3884; $\mathrm{P}<0.01)$, Poaceae sp. $1(\mathrm{R}=0.2619$; $\mathrm{P}<0.01)$ y Bastardia sp. $1(\mathrm{R}=0.1176 ; \mathrm{P}<$ $0.05)$, mientras que E. ruidum solo tuvo preferencia por la semilla Panicum sp. 1 ( $\mathrm{R}=$ 0.1489; $\mathrm{P}<0.05)$.

Experimento de germinación: Se colocó a germinar 895 semillas de las cuales

CUADRO 1

Semillas más abundantes encontradas en los nidos de Pheidole fallax en las épocas y áreas rehabilitadas de la mina de carbón el Cerrejón

TABLE 1

More abundant seeds found in the nests of Pheidole fallax in different seasons, in rehabilitated areas of the Cerrejón coal mine

\begin{tabular}{|c|c|c|c|c|c|c|c|c|c|c|c|}
\hline \multirow{3}{*}{ Semillas } & \multicolumn{3}{|c|}{ Época seca } & \multirow{3}{*}{ Total } & \multicolumn{6}{|c|}{ Época de lluvias } & \multirow{3}{*}{ Total } \\
\hline & \multicolumn{3}{|c|}{20 años } & & \multicolumn{3}{|c|}{9 años } & \multicolumn{3}{|c|}{20 años } & \\
\hline & $\mathrm{N}$ & $\mathrm{C}$ & B & & $\mathrm{N}$ & $\mathrm{C}$ & B & $\mathrm{N}$ & $\mathrm{C}$ & B & \\
\hline Desmanthus sp. 1 & 100 & 4 & 2786 & 2890 & 0 & 0 & 0 & 116 & 0 & 547 & 663 \\
\hline Poaceae sp. 1 & 14 & 7 & 88 & 109 & 0 & 0 & 7 & 254 & 0 & 868 & 1129 \\
\hline Poaceae sp. 2 & 0 & 0 & 0 & 0 & 0 & 0 & 0 & 201 & 0 & 527 & 728 \\
\hline Bastardia sp. 1 & 0 & 0 & 0 & 0 & 34 & 0 & 1447 & 4 & 0 & 4985 & 6470 \\
\hline Panicum sp. 1 & 0 & 0 & 0 & 0 & 0 & 0 & 4 & 0 & 0 & 4411 & 4415 \\
\hline Abundancia & 114 & 11 & 2874 & 2999 & 34 & 0 & 1458 & 575 & 0 & 11338 & 13405 \\
\hline
\end{tabular}

Nido (N), Control (C) y Basurero (B). 

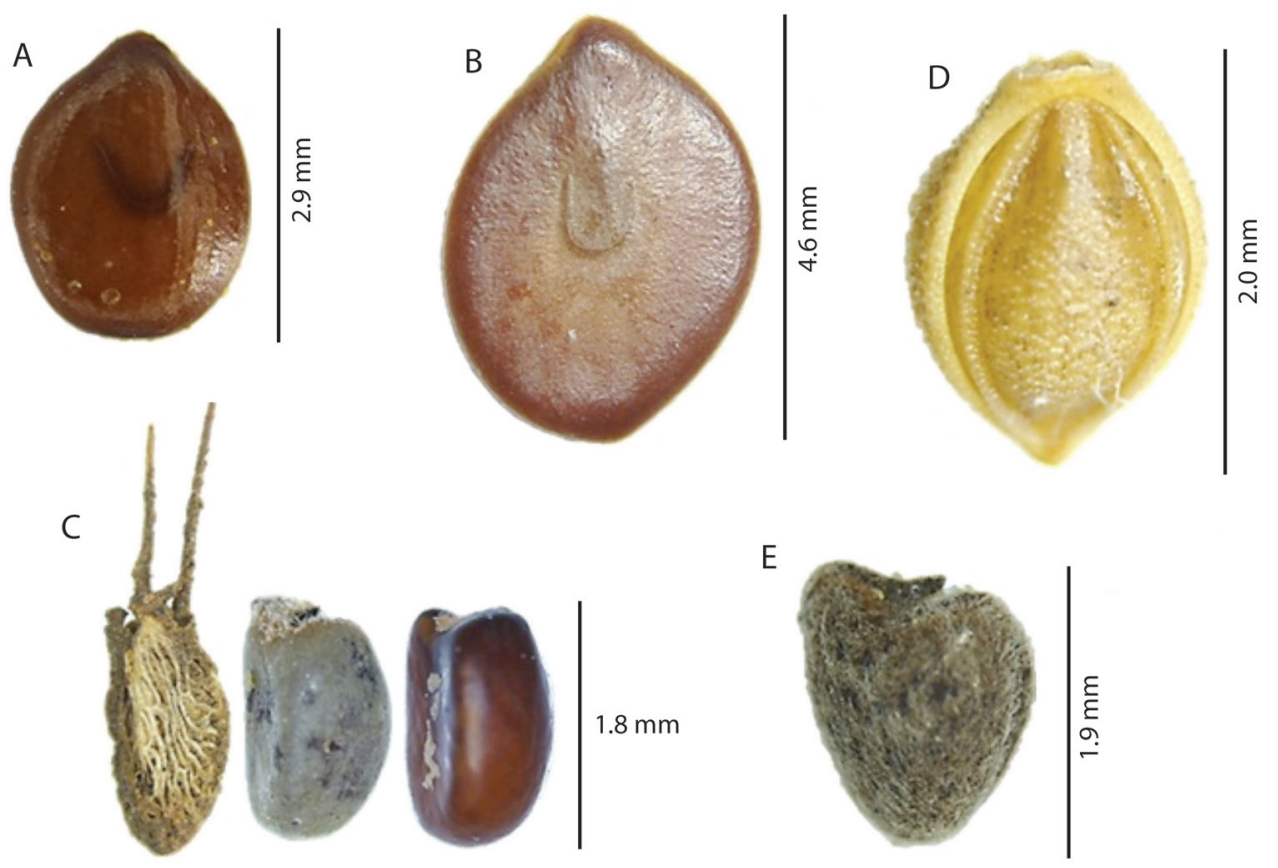

Fig. 3. Semillas más abundantes y frecuentes, encontradas en nidos, basureros de P. fallax y E. ruidum y control. Desmanthus sp. 1 (A), Fabaceae sp. 1 (B), Poaceae sp. 1 (C), Panicum sp. 1 (D), Bastardia sp. 1 (E).

Fig. 3. More abundant and frequent seeds found in nests and dumps of E. ruidum and P. fallax and in nearby control soils. Desmanthus sp. 1 (A), Fabaceae sp. 1 (B), Poaceae sp. 1 (C), Panicum sp. 1 (D), Bastardia sp. 1 (E).

\section{CUADRO 2}

Semillas más abundantes encontradas en los nidos de Ectatomma ruidum en las épocas y áreas rehabilitadas de la mina de carbón el Cerrejón

TABLE 2

More abundant seeds found in the nests of Ectatomma ruidum in different seasons, in rehabilitated areas of the Cerrejón coal mine

\begin{tabular}{|c|c|c|c|c|c|c|c|c|c|c|}
\hline \multirow{3}{*}{ Semillas } & \multirow{2}{*}{\multicolumn{2}{|c|}{$\begin{array}{c}\text { Época seca } \\
\text { Bosque }\end{array}$}} & \multirow{3}{*}{ Total } & \multicolumn{6}{|c|}{ Época de lluvias } & \multirow{3}{*}{ Tota } \\
\hline & & & & \multicolumn{2}{|c|}{9 años } & \multicolumn{2}{|c|}{20 años } & \multicolumn{2}{|c|}{ Bosque } & \\
\hline & $\mathrm{N}$ & $\mathrm{C}$ & & $\mathrm{N}$ & $\mathrm{C}$ & $\mathrm{N}$ & $\mathrm{C}$ & $\mathrm{N}$ & $\mathrm{C}$ & \\
\hline Desmanthus sp. 1 & 0 & 0 & 0 & 0 & 1 & 18 & 1 & 0 & 0 & 20 \\
\hline Fabaceae sp. 1 & 15 & 9 & 24 & 2 & 0 & 5 & 1 & 15 & 3 & 26 \\
\hline Panicum sp. 1 & 0 & 0 & 0 & 0 & 0 & 0 & 0 & 334 & 93 & 427 \\
\hline Abundancia & 15 & 9 & 24 & 2 & 1 & 23 & 2 & 349 & 96 & 473 \\
\hline
\end{tabular}

Nido (N), Control (C) y Basurero (B).

germinaron $235(26.3 \%)$. Para las semillas extraídas de los nidos de E. ruidum germinó un $74.5 \%$ frente a un $25.5 \%$ de semillas tomadas de los controles. La prueba de U-MannWhitney determinó que existen diferencias significativas en cuanto a la germinación $(\mathrm{U}=$ 22, $\mathrm{P}<0.01$ ).

En el caso de $P$. fallax los porcentajes de germinación en basureros $(57.4 \%)$ y nidos $(41 \%)$ fueron mayores a los observados 
CUADRO 3

Semillas que presentaron mayor porcentaje de germinación en nidos, suelos control y basureros de P. fallax

TABLE 3

Seeds that have a higher percentage of germination in nests, control soils and $P$. fallax dumps

\begin{tabular}{|c|c|c|c|c|c|c|c|}
\hline \multirow{2}{*}{ Semilla } & \multicolumn{2}{|c|}{ Nidos } & \multicolumn{2}{|c|}{ Control } & \multicolumn{2}{|c|}{ Basureros } & \multirow{2}{*}{$\begin{array}{l}\text { Porcentaje de } \\
\text { Germinación }\end{array}$} \\
\hline & $\mathrm{N}$ & G & $\mathrm{N}$ & $\mathrm{G}$ & $\mathrm{N}$ & G & \\
\hline Desmanthus sp. 1 & 163 & 33 & 0 & 0 & 147 & 39 & 43.6 \\
\hline Bastardia sp. 1 & 10 & 6 & 0 & 0 & 65 & 21 & 16.4 \\
\hline Panicum sp. 1 & 5 & 2 & 0 & 0 & 32 & 19 & 12.7 \\
\hline Fabaceae sp. 1 & 60 & 27 & 9 & 3 & 34 & 15 & 27.3 \\
\hline Total & 238 & 68 & 9 & 3 & 278 & 94 & 100 \\
\hline
\end{tabular}

Número de semillas puestas a germinar $(\mathrm{N})$, semillas germinadas $(\mathrm{G})$.

en el control (1.6\%). La prueba de KruskalWallis determinó que existen diferencias significativas en cuanto a la germinación entre muestras $(H=12.92, P<0.01)$. La germinación en el control fue significativamente menor que la observada en el basurero $(\mathrm{U}=11, \mathrm{P}<0.01)$ y el nido $(\mathrm{U}=30, \mathrm{P}<0.01)$, la tasa de germinación fue similar entre nidos y basureros $(\mathrm{U}=$ $56, \mathrm{P}>0.05)$.

Respecto a las semillas más abundantes $\mathrm{y}$ frecuentes encontradas en nido, control y basurero de $P$. fallax, las semillas que presentaron mayor porcentaje de germinación fueron Desmanthus sp. 1, Fabaceae sp. 1, Bastardia sp. 1 y Panicum sp. 1 (Cuadro 3). Panicum sp. 1 presentó el mayor porcentaje de germinación en nido y control de E. ruidum, los demás tipos de semillas presentaron valores muy bajos (Cuadro 3).

Interacción hormigas-semillas: Para Pheidole fallax en el primer experimento, las hormigas siempre removieron la semilla de menor tamaño (1.2 a $2.5 \mathrm{~mm}$ ). En la mayoría de los eventos las semillas fueron llevadas al tubo nido (50\%), en ocasiones fueron abandonadas en el camino (35\%) o eran consumidas en el mismo lugar $(15 \%)$. En el segundo experimento, se usó la semilla de Desmanthus sp. 1 y se observaron tres comportamientos: 1) las semillas fueron removidas por obreras de esta especie al tubo-nido donde la manipularon, la consumieron y los desechos fueron depositados en el basurero, 2) las semillas fueron acumuladas dentro del tubo-nido y no manipuladas y 3) las semillas introducidas al tubo-nido después de unos días fueron sacadas intactas al basurero debido a la acumulación de semillas dentro del tubo-nido.

En el tercer experimento, las obreras removieron la semilla y la ingresaron al tubo-nido, allí retiraron la parte carnosa la cual fue fraccionada en pedazos más pequeños, que luego fueron consumidos por obreras y soldados. Después de retirar la parte carnosa, la semilla intacta fue depositada en el basurero.

Para Ectatomma ruidum en el primer experimento las hormigas de esta especie removieron las semillas de menor tamaño, llevándolas al tubo-nido (40\%), en ocasiones las abandonaron en el camino (35\%) o eran consumidas en el mismo lugar (25\%). En el segundo experimento se ofrecieron semillas de Fabaceae sp. 1. Se evidenciaron tres comportamientos. 1) Las obreras reconocieron la semilla y la removieron al interior del tubo-nido, 2) las hormigas removieron la semilla y fue abandonada en el camino y 3) Las semillas fueron inspeccionadas pero no removidas por ninguna de las obreras de esta especie. En el caso de la semilla de Capparis sp., las hormigas removieron la semilla cerca del tubo-nido y retiraron la parte carnosa, la cual llevaron dentro del nido y abandonaron la semilla intacta. 


\section{DISCUSIÓN}

Las especies tienen un aporte diferencial en la remoción de semillas en las áreas de restauración de la mina, mientras que Pheidole fallax impacta más hacia la edad de veinte años, Ectatomma ruidum lo hace en las áreas de nueve años. Se resalta que las semillas removidas por ambas especies pertenecen a plantas diferentes, lo cual podría favorecer el restablecimiento de la capa vegetal de las áreas en rehabilitación, tal como lo han demostrados varios estudios realizados por Pizo y Oliveira (2000), Giladi (2006), Christianini et al. (2007) y Leal et al. (2007) los cuales reportan que las semillas removidas y transportadas por las hormigas a sus nidos, basureros o áreas vecinas, presentan una mayor tasa de germinación y establecimiento, lo que podría ayudar a la recuperación de la capa vegetal.

Pese a que ambas especies de hormigas realizan la función de dispersar semillas en diferentes áreas de rehabilitación, E. ruidum remueve menos semillas que $P$. fallax, sólo el $1.5 \%$ del total de semillas encontradas. Este resultado se debe a la preferencia trófica de esta especie, la cual se destaca principalmente por ser depredadora de pequeños invertebrados, un gran porcentaje de la dieta de esta especie puede estar compuesta por insectos de los órdenes Coleoptera, Dermaptera, Diptera, Hymenoptera e Isoptera (Riera-Valera \& Pérez-Sánchez 2009). Resultados similares fueron encontrados por Santamaría et al. (2009 a) quien reporta que esta especie presenta una dieta que incluye un fuerte componente de garrapatas (Rhipicephalus microplus). Lo observado en esta investigación podría dejar evidencia de que la dispersión de semillas por esta hormiga, obedecería más a una dieta secundaria, la cual estaría sujeta a la disponibilidad de recurso en el área. Estos resultados no difieren de lo encontrado en esta investigación, donde si bien no fueron cuantificados, se observó un alto volumen de restos de insectos como Coleoptera e Hymenoptera.

$P$. fallax remueve grandes cantidades de semillas en las áreas donde se encuentra asociada (Wilson, 2003; Zelikova \& Breed, 2008; Domínguez-Haydar \& Armbrecht, 2011; Lôbo et al., 2011), en esta investigación se destaca que en solo 15 nidos con sus respectivos basureros fueron extraídas 20349 semillas, lo que demuestra que esta hormiga presenta una dieta con un fuerte componente de semillas o de estructuras accesorias como eleosoma o arilos (Lôbo et al., 2011), es probable que la alta acumulación de semillas en sus nidos obedezca a una estrategia para mantener recurso disponible para su alimentación (Levey \& Byrne, 1993; Pizo, 2008).

Las hormigas remueven en su mayoría semillas de plantas herbáceas presentes en cada una de las zonas (Redolfi, Tinaut, Pascual, \& Campos, 2004; Giladi, 2006). Es posible que la mayor remoción de semillas de herbáceas obedezca a dos razones, la primera es la dominancia de este estrato vegetal en las primeras etapas de la restauración, dado que el suelo es estabilizado con pastos y se produce la colonización por las plantas pioneras, las cuales en su mayoría son herbáceas y serían las que principalmente aportan las semillas (Redolfi et al., 2004).

La segunda razón por la que las hormigas remueven mayormente semillas de herbáceas puede estar relacionada con el tamaño de la semilla, característica que ha sido descrita como un factor que facilita o limita la dispersión de semillas (Oliveira, Galetti, Pedroni, \& Morellato, 1995; Pizo \& Oliveira, 2001; Gomez, Espadaler, \& Bas, 2005; Dunlop \& Rodgerson, 2006). Esto fue corroborado en el experimento de interacción donde se demostró que tanto $P$. fallax como E. ruidum removieron mayormente semillas de tamaños pequeños, en el área de estudio estas semillas de herbáceas tenían entre 1.3-6.8 $\mathrm{mm}$ y un peso promedio de 0.003 gr. No obstante las hormigas del género Pheidole recurren al reclutamiento masivo de obreras para remover la semilla al nido en el caso que estas sean grandes para ser removida por una hormiga (Itzkowitz \& Haley, 1983; Escobar, Armbrecht, \& Calle, 2007). En otros casos donde las semillas son grandes y con un gran eleosoma, arilo o pulpa carnosa rica 
en lípidos o ácidos grasos, las hormigas optan por retirar esta parte y dejan la semilla intacta en el sitio donde fue encontrada (Pizo \& Oliveira, 2001; Passos \& Oliveira, 2003; Christianini \& Oliveira, 2010).

Respecto a E. ruidum Escobar et al. (2007) reportan que esta hormiga remueve mayormente semillas de Passiflora ligularis (semillas de tamaño pequeño $5-8 \mathrm{~mm}$ ), resultados que también fueron reportados por Santamaría et al. (2009 a) para hábitats similares. El tamaño de la semilla y la presencia de arilo pueden favorecer la remoción de semillas pequeñas ya que atraen una mayor cantidad de dispersores (Escobar et al. 2007). Por otro lado Pizo y Oliveira (2001) y Leal (2003) proponen que este tipo de diáspora es fácil de remover por un solo individuo de tamaño mediano como E. ruidum, sin la necesidad de reclutamiento de más obreras, lo que reduce consecuentemente la exposición de la hormiga a potenciales depredadores (Gomez et al., 2005). Por otra parte es probable que la preferencia de semillas pequeñas por parte $E$. ruidum obedezca también a la arquitectura de sus nidos, ya que estos presentan un tamaño de entrada de 5mm (Medina, 1994), lo cual limitaría a esta hormiga a un forrajeo de semilla de tamaño similar o inferior a este diámetro.

Las semillas alojadas en los nidos y basureros de las hormigas, tuvieron un mayor éxito de germinación, los nidos son reconocidos como lugares que ofrecen condiciones estables que evitan el deterioro de las diásporas, lo que permite que estas mantengan su viabilidad por un tiempo prolongado (Pizo \& Oliveira, 2001; Leal, 2003; Christianini et al., 2007; Leal et al., 2007).

Otros beneficio que las hormigas aportan a las semillas consiste en el retiro de ciertas cubiertas como el arilo y el eleosoma (Christianini et al., 2007), ya que deja al descubierto el micrópilo, que permite que el agua y el oxígeno ingresen a la semilla y que esta salga del estado de latencia y tenga un mayor éxito de germinación (Leal et al., 2007). La remoción de estas estructuras también evita la infección por hongos, por ejemplo Oliveira et al. (1995) observaron que las semillas de Hymenaea courbaril cuya parte carnosa fue retirada por la hormiga Mycocepurus goeldii, presentaron un mayor éxito de germinación y no fueron infectadas por hongos.

Los altos valores de germinación de semillas observados en los nidos de ambas especies de hormigas son similares a los reportados por García-Robledo y Kuprewicz (2009), para E. ruidum, los cuales encontraron que las semillas de Renealmia alpinia, removidas al nido y desprovistas del arilo tuvieron un éxito de germinación mayor que las que no fueron manipuladas por E. ruidum. Para el caso de P. fallax no se reporta en la literatura información acerca de su contribución en la germinación de semillas, sin embargo, autores como Levey y Byrne (1993), encontraron que las semillas en los nidos de dos especies del género Pheidole ( $P$. nigricula y $P$. nebulosre), presentaron mayor germinación que las semillas encontradas en los basureros y controles (vea también Pizo, 2008). Por otra parte, las semillas encontradas en los controles germinaron en mayor proporción que las de los basureros. Sin embargo, las semillas germinadas en basureros lograron establecerse en mayor cantidad que las de nido y control.

Por último, la mayor parte de las semillas encontradas en los nidos de ambas especies de hormigas pertenecen a las familias Fabaceae, Malvaceae y Poaceae. La alta germinación de estas leguminosas en los nidos de estas hormigas puede ser un buen indicador de la rehabilitación de las áreas degradadas, ya que las especies de la familia Fabaceae se consideran excelentes fijadoras de nitrógeno y aportan gran cantidad de materia orgánica al suelo, lo que facilita el establecimiento de otras plantas (Armstrong, Kuskopf, Millar, Whitbread, \& Standley, 1999; Figueroa \& Galeano, 2007). En particular, se ha encontrado que plantas del género Desmanthus aumentaron el carbono orgánico y el nitrógeno en la capa superior del suelo (Armstrong et al., 1999).

Nuestros resultados sugieren que tanto las semillas depositadas en nidos de E. ruidum como en nidos y basureros de $P$. fallax que no son consumidas, son semillas con alta 
posibilidad de germinación, lo que podría contribuir a la recuperación de la capa vegetal de las áreas de rehabilitación donde se encuentren estas dos especies de hormigas. Sería importante explorar si $P$. fallax y E. ruidum modifican las propiedades físicas y químicas de los suelos a través de la construcción de los nidos y en términos generales, en áreas bajo procesos de restauración se recomienda aplicar modelos que permitan evaluar la contribución de la biodiversidad a la recuperación de interacciones y funciones ecosistémicas (Montoya, Rogers, \& Memmott, 2012).

\section{AGRADECIMIENTOS}

Los autores desean agradecer al Departamento Administrativo de Ciencia, Tecnología e Innovación, COLCIENCIAS y a la Universidad del Atlántico por la financiación de este proyecto (código 1116-569-34827). Al personal administrativo de la mina del Cerrejón. A los semilleros de investigación de Restauración Ecológica y en Insectos (NEOPTERA) del Caribe colombiano, a Kevin Álvarez y Jorge Luis Rangel Acosta por el apoyo en laboratorio y durante el proyecto y a Roberto Guerrero por su ayuda en la determinación taxonómica de las hormigas.

\section{RESUMEN}

La dispersión de semillas es un proceso clave en el restablecimiento de la vegetación en áreas rehabilitadas. El objetivo de este trabajo fue determinar la contribución de las hormigas Ectatomma ruidum y Pheidole fallax en la dispersión y germinación de semillas en áreas rehabilitadas en la mina de carbón del Cerrejón, Colombia. En cada área se instalaron cuatro parcelas de 6 × $40 \mathrm{~m}$, donde se contó el número de nidos por especie y se midió la distancia entre los nidos más próximo de cada especie. De los nidos contabilizados cinco nidos fueron seleccionados para la extracción de semillas, por cada nido extraído se tomó un control. Un total de 295 nidos fueron contabilizados, 59 para la época seca y 236 para la época de lluvia, 84 pertenecían a $P$. fallax y 211 a E. ruidum. Un total de 21304 semillas fueron encontradas en los nidos y suelos control, 19349 fueron obtenidas de los nidos y basureros de $P$. fallax y 318 en nidos de E. ruidum, en el suelo control se hallaron 597 semillas. El número de semillas extraídas en los nidos fue significativamente mayor a la de los controles. El número de semillas removidas por $P$. fallax presentó diferencias significativas respecto a las removidas por E. ruidum. El porcentaje de germinación en los nidos fue mayor que en los controles, en el caso de $P$. fallax, el porcentaje de germinación fue bajo en los controles y alto tanto en los basureros como los nidos. Nuestros resultados sugieren que $E$. ruidum y $P$. fallax podrían contribuir a la dispersión de las semillas y al restablecimiento de la vegetación en las áreas donde se encuentran, lo cual es un aporte significativo para la rehabilitación de las áreas degradas por la minería.

Palabras clave: mirmecocoria, nidos, restauración, basureros, propágulos.

\section{REFERENCIAS}

Armstrong, R. D., Kuskopf, B. J., Millar, G., Whitbread, A. M., \& Standley, J. (1999). Changes in soil chemical and physical properties following legumes and opportunity cropping on a cracking clay soil. Animal Production Science, 39, 445-456.

Beattie, A. J. (1985). The evolutionary ecology of ant-plant mutualisms. Cambridge: Cambridge University Press.

Beattie, A. (1989). The effects of ants on grasslands. In L. F. Huenneke, \& H. A. Mooney (Eds.), Grassland structure and function (pp. 105-116). USA: Springer.

Breed, M. D., Abel, P., Bleuze, T. J., \& Scott, E. D. (1990). Thievery, home ranges, and nestmate recognition in Ectatomma ruidum. Oecologia, 84, 117-121.

Breed, M. D., McGlynn, T. P., Stocker, E. M., \& Klein, A. N. (1999). Thief workers and variation in nestmate recognition behavior in a ponerine ant, Ectatomma ruidum. Insectes Sociaux, 46, 327-331.

Christianini, A. V., Mayhé-Nunes, A. J., \& Oliveira, P. S. (2007). The role of ants in the removal of nonmyrmecochorous diasporas and seed germination in a neotropical savanna. Journal of Tropical Ecology, 23, 343-351.

Christianini, A. V., \& Oliveira, P. S. (2010). Birds and ants provide complementary seed dispersal in a neotropical savanna. Journal of Ecology, 98, 573-582.

Christianini, A. V., Mayhé-Nunes, A. J., \& Oliveira, P. S. (2012). Exploitation of Fallen Diaspores by Ants: Are there Ant-Plant Partner Choices? Biotropica, $44,360-367$.

Delabie, J. H. C., Ospina, M., \& Zabala, G. (2003). Relaciones entre hormigas y plantas: una introducción. En F. Fernández (Ed.), Introducción a las Hormigas de la Región Neotropical (Capítulo 10, pp. 167-180). Bogotá, Colombia: Instituto de Investigación de Recursos biológicos Alexander von Humboldt Bogotá. 
Domínguez-Haydar, Y., \& Armbrecht, I. (2011). Response of ants and their seed removal in rehabilitation areas and forests at El Cerrejón coal mine in Colombia. Restoration Ecology, 19, 178-184.

Dufrene, M., \& Legendre, P. (1997). Species Assemblages and Indicator Species: The Need for a Flexible Asymmetrical Approach. Ecological Monographs, 67, 345-366.

Escobar, S., Armbrecht, I., \& Calle, Z. (2007). Transporte de semillas por hormigas en bosques y agroecosistemas ganaderos de los Andes Colombianos. Agroecología, 2, 65-74.

Escobar-Ramírez, S., Duque, S., Henao, N., Hurtado-Giraldo, A., \& Armbrecht, I. (2012). Removal of Nonmyrmecochorous Seeds by Ants: Role of Ants in Cattle Grasslands. Psyche: A Journal of Entomology, 1-8.

Farnese, F. S., Mângia, S., \& Fonseca, G. A. (2011). Mirmecocoria: uma revisão. Revista Eletrônica de Biologia, 3, 53-65.

Figueroa, Y., \& Galeano, G. (2007). Lista comentada de las plantas vasculares del enclave seco interandino de $\mathrm{La}$ Tatacoa (Huila, Colombia). Caldasia, 29, 263-281.

García-Robledo, C., \& Kuprewicz, E. K. (2009). Vertebrate Fruit Removal and Ant Seed Dispersal in the Neotropical Ginger Renealmia alpinia (Zingiberaceae). Biotropica, 41, 209-214.

Giladi, I. (2006). Choosing benefits or partners: a review of the evidence for the evolution of myrmecochory. Oikos, 112, 481-492.

Gomez, C., Espadaler, X., \& Bas, J. M. (2005). Ant behaviour and seed morphology: a missing link of myrmecochory. Oecologia, 146, 244-246.

Gualdrón, R. (2011). Hacia la rehabilitación de las tierras intervenidas por la minería a cielo abierto. Colombia: Cerrejón.

Hammer, Ø. (2015). PAleontological STatistics (PAST) (Versión 3.10). Recuperado de http://folk.uio.no/ ohammer/past/

Howe, H. F., \& Smallwood, J. (1982). Ecology of seed dispersal. Annual Review of Ecology and Systematics, 13, 201-228.

Itzkowitz, M., \& Haley, M. (1983). The food retrieval tactics of the ant Pheidole fallax Mayr. Insectes Sociaux, 30, 317-322.

Jaimes, G. \& Moreno, M. (2014). Componente botánico. En L. Báez \& F. Trujillo (Eds.), Biodiversidad en Cerrejón (pp. 57-81). Bogotá, Colombia: Carbones de Cerrejón, Fundación Omacha, Fondo para la Acción Ambiental y la Niñez.
Leal, I. R. (2003). Dispersão de sementes por formigas na Caatinga. En I. R. Leal, M. Tabarelli, \& J. M. Cardoso (Eds.), Ecologia e Conservacao da Caatinga (pp. 593-624). Brasil: Universidade Federal de Pernambuco.

Leal, I. R., Wirth, R., \& Tabarelli, M. (2007). Seed dispersal by ants in the semi-arid Caatinga of north-east Brazil. Annals of Botany, 99, 885-894.

Levey, D. J., \& Byrne, M. M. (1993). Complex ant-plant interactions: rain-forest ants as secondary dispersers and post-dispersal seed predators. Ecology, 74, 1802-1812.

Lôbo, D., Tabarelli, M., \& Leal, I. R. (2011). Relocation of Croton sonderianus (Euphorbiaceae) seeds by Pheidole fallax Mayr (Formicidae): a case of postdispersal seed protection by ants? Neotropical Entomology, 40, 440-444.

Medina, C. (1994). Nidificación y patrones de distribución espacial de nidos de hormigas en una sabana tropical, Carimagua: LLanos Orientales de Colombia. Boletín del Museo de Entomología de la Universidad del Valle, 2, 31-42.

Montoya, D., Roge, L., \& Memmott, J. (2012). Emerging perspectives in the restoration of biodiversity-based ecosystem services. Trends in Ecology and Evolution, 27, 666-672.

Oliveira, P. S., Galetti, M., Pedroni, F., \& Morellato, P. (1995). Seed cleaning by Mycocepurus goeldii ants (Attini) facilitates germination in Hymenaea courbaril (Caesalpiniaceae). Biotropica, 27, 518-522.

Passos, L., \& Oliveira, P. S. (2003). Interactions between ants, fruits and seeds in a restinga forest in south-eastern Brazil. Journal of Tropical Ecology, 19, 261-270.

Pizo, M. A., \& Oliveira, P. S. (2000). The Use of Fruits and Seeds by Ants in the Atlantic Forest of Southeast Brazil 1. Biotropica, 32, 851-861.

Pizo, M. A., \& Oliveira, P. S. (2001). Size and lipid content of nonmyrmecochorous diaspores: effects on the interaction with litter-foraging ants in the Atlantic rain forest of Brazil. Plant Ecology, 157, 37-52.

Pizo, M. A., Passos, L., \& Oliveira, P. S. (2004). Ants as seed dispersers of fleshy diaspores in Brazilian Atlantic forests. En P. M. Forget, J. E. Lambert., P. E. Hulme, \& S. B Vander-Wall (Eds.), Seed fate: Predation and secondary dispersal. Wallingford: CABI Publishing (pp. 315-329). Wallingford, Oxfordshire: CABI Publishing.

Pizo, M. A. (2008). The Use of Seeds by a Twig-Dwelling Ant on the Floor of a Tropical Rain Forest. Biotropica, 40, 119-121.

R Development Core Team. (2014). R a language and environment for statistical computing (Versión 3.1.1). 
Vienna, Austria: R foundation for Statistical Computing. Recuperado de http://www.R-project.org/

Redolfi, I., Tinaut, A., Pascual, F., \& Campos, M. (2004). Densidad de nidos de la comunidad de hormigas (Formicidae) en tres olivares con diferente manejo agronómico en Granada, España. Ecología Aplicada, 3, 73-81.

Riera-Valera, M., \& Pérez-Sánchez, A. J. (2009). Notas acerca de la dieta de Ectatomma ruidum (Roger 1861) (Hymenoptera: Formicidae: Ectatomminae) en un jardín venezolano. Boletín Sociedad Entomológica Aragonesa, 44, 550-552.

Salas, J. S., Ybarra, E. J., Moreno, M. P., Rivas, J. F., \& Pérez, G. M. (2010). Estrategias germinativas de las semillas en ambientes áridos. Revista Chapingo Serie Zonas Áridas, 9, 35-38.

Santamaría, C., Armbrecht, I., \& Lachaud, J. P. (2009a). Nest Distribution and Food Preferences of Ectatomma ruidum (Hymenoptera: Formicidae) in Shaded and Open Cattle Pastures of Colombia. Sociobiology, 53, 517-541.
Santamaría, C., Domínguez Haydar, Y., \& Armbrecht, I. (2009b). Cambios en la distribución de nidos y abundancia de la hormiga Ectatomma ruidum (Roger 1861) en dos zonas de Colombia. Boletín del Museo de Entomología de la Universidad del Valle, $10,10-18$.

Torres, G., Zapote, C. A., \& López, F. (2013a). Guía técnica para el reconocimiento y la identificación de semillas de maleza en pastos de Brasil. México: Secretaría de Agricultura, Ganadería, Desarrollo rural, Pesca y Alimentación.

Torres, G., Zapote, C. A., \& López, F. (2013b). Guía técnica para la identificación de semillas de malezas en Alpiste. México: Secretaría de Agricultura, Ganadería, Desarrollo Rural, Pesca y Alimentación.

Wilson, E. O. (2003). Pheidole in the New World: a dominant, hyperdiverse ant genus. London, England: Harvard University Press.

Zelikova, T. J., \& Breed, M. D. (2008). Effects of habitat disturbance on ant community composition and seed dispersal by ants in a tropical dry forest in Costa Rica. Journal of Tropical Ecology, 24, 309-316. 
